\title{
Correction to: Influence of fellow eye on the diagnosis and classification of central serous chorioretinopathy
}

\author{
Deepika C. Parameswarappa ${ }^{1}$. Supriya Arora ${ }^{2} \cdot$ Sumit Randhir Singh ${ }^{3} \cdot$ Niroj Kumar Sahoo $^{4} \cdot$ Dmitrii S. Maltsev $^{5}$. \\ Alexei N. Kulikov ${ }^{5} \cdot$ Claudio lovino $^{6} \cdot$ Filippo Tatti $^{7} \cdot$ Ramesh Venkatesh $^{8} \cdot$ Haniah Zaheer $^{9} \cdot$ Nikitha Gurram Reddy $^{8}$. \\ Ram Snehith Pulipaka ${ }^{8} \cdot$ Enrico Peiretti $^{7} \cdot$ Jay Chhablani $^{9,10}$
}

Published online: 11 November 2021

๑) Springer-Verlag GmbH Germany, part of Springer Nature 2021

\section{Correction to: Graefe's Archive for Clinical and Experimental Ophthalmology https://doi.org/10.1007/s00417-021-05435-2}

In the original published article, one of the author names has been misspelled.

"Ramesh Vankatesh" should be "Ramesh Venkatesh"

This is being corrected in this publication.

Publisher's note Springer Nature remains neutral with regard to jurisdictional claims in published maps and institutional affiliations.

The original article can be found online at https://doi.org/10.1007/ s00417-021-05435-2.

Jay Chhablani

jay.chhablani@gmail.com

1 Smt Kanuri Santhamma Center for Vitreo-Retina Diseases, L V Prasad Eye Institute, Hyderabad, Telangana, India

2 Division of Ophthalmology, Department of Surgery, Princess Margaret Hospital, Nassau, Bahamas

3 Jacobs Retina Center at Shiley Eye Center, University of California, San Diego, La Jolla, CA, USA

4 Department of Retina and Vitreous, L V Prasad Eye Institute, Vijayawada, India

5 Department of Ophthalmology, Military Medical Academy, St. Petersburg, Russian Federation
6 Eye Clinic, Multidisciplinary Department of Medical, Surgical and Dental Sciences, University of Campania Luigi Vanvitelli, Naples, Italy

7 Department of Surgical Sciences, Eye Clinic, University of Cagliari, Cagliari, Italy

8 Department of Retina and Vitreous, Narayana Nethralaya, Bengaluru, India

9 Department of Ophthalmology, University of Pittsburgh Eye and Ear Institute, Pittsburgh, PA, USA

10 UPMC Eye Center, University of Pittsburgh, Pittsburgh, PA, USA 